\title{
SCIENTIFIC REPORTS

\section{OPEN Recurrent gene duplication in the angiosperm tribe Delphinieae (Ranunculaceae) inferred from intracellular gene transfer events and heteroplasmic mutations in the plastid matK gene}

\author{
Seongjun Park $\mathbb{D}^{1,2}$, Boram $A n^{2} \&$ SeonJoo Park ${ }^{2 *}$
}

The study of intracellular gene transfer may allow for the detection of interesting evolutionary processes such as ancient polyploidization. We compared 24 plastid genomes (plastomes) from tribe Delphinieae, one from tribe Nigelleae and one from tribe Ranunculeae, including five newly sequenced genomes. The functional transfers of the plastids rpl32 and rps16 to the nucleus in tribe Delphinieae were identified. Unexpectedly, we discovered multiple divergent copies of the nuclear-encoded plastid $r p l 32$ in the genus Aconitum. Phylogenetic and synonymous substitution rate analyses revealed that the nuclear-encoded plastid $r p l 32$ underwent two major duplication events. These ancient gene duplication events probably occurred via multiple polyploidization events in Aconitum between 11.9 and 24.7 Mya. Furthermore, our sequence rate analysis indicated that the eight plastid-encoded $r p l$ subunits in Aconitum had a significantly accelerated evolutionary rate compared to those in other genera, suggesting that highly divergent paralogs targeted to the plastid may contribute to an elevated rate of evolution in plastid $r p l$ genes. In addition, heteroplasmy of the plastid matK from two Aconitum species suggested the existence of potentially functional plastid maturases in its plastome. Our results provide insight into the evolutionary history of the tribe Delphinieae.

Gene duplication (GD) has played an important role in eukaryotic evolution by generating evolutionary novelty ${ }^{1}$. GD occurs through various mechanisms, including whole-genome duplication (WGD), tandem duplication, transposon-mediated duplication, segmental duplication, and retroduplication ${ }^{2}$. After a duplication event, the duplicated genes are usually functionally redundant, resulting in pseudogenization and gene loss ${ }^{3}$. However, if the presence of duplicated genes is beneficial, the copies may acquire new functions via subfunctionalization ${ }^{4}$ and neofunctionalization ${ }^{1}$. Furthermore, duplicated copies can be retained without acquiring novel functions due to gene dosage effects 5 . In plants, WGD or polyploidization provides a notable opportunities for adaptive radiation, speciation and diversification ${ }^{6-9}$. Many plant lineages, such as Brassicaceae, Fabaceae, Poaceae, and Solanaceae, have undergone one or more genome-doubling events, leading to increased species richness ${ }^{10}$. Detecting and evaluating WGDs in plants is challenging because of rapid gene losses or diploidization over time $\mathrm{e}^{11,12}$. Moreover, recurrent WGDs during plant evolution can promote chromosomal rearrangement ${ }^{13,14}$, which complicates the identification of events.

The tribe Delphinieae Schröd. is one of the largest tribes within Ranunculaceae; it comprises $>650$ species, accounting for $\sim 26 \%$ of all Ranunculaceae species ${ }^{15}$. The species in this tribe exhibit anatomical and morphological diversity in their flowers ${ }^{16,17}$. Polyploidization has played a critical role in the speciation and diversification of tribe Delphiniea $e^{18}$. This tribe exhibits an enormous range of polybasic chromosome numbers $(x=6$, $7,8,9,10$, and 13 ; main basic number $\mathrm{x}=8)$, and ploidy levels from diploid $(2 \mathrm{n}=12)$ to octoploid $(2 \mathrm{n}=64)^{19}$.

${ }^{1}$ Institute of Natural Science, Yeungnam University, Gyeongsan, Gyeongbuk, 38541, South Korea. ${ }^{2}$ Department of Life Sciences, Yeungnam University, Gyeongsan, Gyeongbuk, 38541, South Korea. *email: sjpark01@ynu.ac.kr 
Moreover, many Delphinieae species, especially those of the genus Aconitum, form species complexes by producing interspecific hybrids in their natural habitat ${ }^{20}$. Thus, this tribe offers an intriguing system to study the dynamics of WGD, including GD, but the understanding of WGD within Delphinieae remains limited. To date, 25 complete Delphinieae plastomes have been sequenced, mainly from the genus Aconitum (23), one from the genus Consolida, and one from the genus Gymnaconitum. The sequenced Delphinieae plastomes generally range in size from 155.5 to $157.4 \mathrm{~kb}$ with a quadripartite organization. Losses of translation initiation factor A (infA) and two plastid-encoded ribosomal protein genes ( $r p l 32$ and rps16) have been documented in the 25 completely sequenced Delphinieae plastomes ${ }^{21-23}$. In angiosperms, intracellular gene transfer (IGT) to the nucleus is an ongoing evolutionary process ${ }^{24}$ and is associated with the pseudogenization or loss of plastid coding genes. The IGT of infA and rpl32 from the plastid to the nucleus has been documented multiple times across angiosperms ${ }^{25-27}$. However, in the case of $r p s 16$, the nuclear-encoded plastid $r p s 16$ was supplanted by a nuclear-encoded mitochondrial rps 16 that had previously been transferred from the mitochondria to the nucleus via $\mathrm{IGT}^{28}$. In addition to gene losses, a truncated plastid-encoded mat $K$ lacking its C-terminal portion has been documented in the $A$. austrokoreense and $A$. chiisanense plastomes ${ }^{29}$. The truncation of protein-coding genes can lead to impairment or loss of gene function, but the function of this gene has not been evaluated.

The study of IGT events can help determine the evolutionary history of GD within tribe Delphinieae. In this study, we generated complete plastome sequences for five species (Aconitum pseudolaeve, Consolida orientalis, Delphinium maackianum, Staphisagria macrosperma, and Nigella damascena) to improve plastome sampling in tribe Delphinieae. Combining these data with previously published plastomes, we examined the phylogenetic distribution of gene losses and identified functional transfers to the nucleus via IGT or gene substitution. These surveys revealed multiple copies of the nuclear-encoded rpl32 among Aconitum species, suggesting at least one GD event in the genus. We analyzed the distributions of synonymous substitution rates $\left(d_{\mathrm{S}}\right)$ among the paralogs of 10 Aconitum species to estimate the number of GD events. The evolutionary fates of the duplicated copies and the potential effects on nucleotide substitution were also evaluated. In addition, we evaluated the functional properties of the truncated plastid-encoded matK gene.

\section{Results}

General features of new plastome sequences. The complete plastomes for four species (A. pseudolaeve, C. orientalis, D. maackianum, and S. macrosperma) from tribe Delphinieae and N. damascena from tribe Nigelleae were determined by paired-end Illumina reads with deep coverages, ranging from $372 \mathrm{x}$ to $1,004 \mathrm{x}$ (Supplementary Table S1 and Supplementary Fig. S1). Each of the five plastomes was assembled into a circular molecule consisting of a large single copy (LSC) and a small single copy (SSC) region separated by a pair of inverted repeats (IRs), and the plastome sizes ranged from $154,484 \mathrm{bp}$ to $155,915 \mathrm{bp}$. In tribe Delphinieae, the four plastomes shared a set of genes that encoded 77 proteins, 30 tRNAs, and 4 rRNAs. The ribosomal protein L32 (rpl32) and the ribosomal protein S16 (rps16) appeared to have been lost or pseudogenized in the plastomes of all four species (Supplementary Fig. S1A). In the case of N. damascena, rpl32 was also lost, but the rps16 gene was an intact gene in its plastome. A contraction at the $\mathrm{IR}_{\mathrm{B}} / \mathrm{SSC}$ boundary resulted in a translocation of $y c f 1$ to the SSC region, and an $\mathrm{IR}_{\mathrm{A}} / \mathrm{SSC}$ expansion included the C-terminal portion of $n d h F(59 \mathrm{bp})$, generating a truncated $n d h F$ fragment in $\mathrm{IR}_{\mathrm{B}}$ (Supplementary Fig. S1B).

Potential functional transfer of two plastid genes to the nucleus in tribe delphinieae. To understand the evolutionary history of gene losses in tribe Delphinieae, we reconstructed phylogenetic relationships and estimated divergence times. The phylogenetic tree, which was inferred from 77 plastid genes with 67,650 aligned nucleotide positions, provided strong support for relationships within tribe Delphinieae (Supplementary Fig. S2). Our analysis suggests that the split between Aconitum and the other genera occurred at 24.7 Mya (95\% highest posterior density (HPD) $=14.49-34.88$; Supplementary Fig. S3). Within the genus Aconitum, the subgenera Aconitum and Lycoctonum diverged at $11.9(95 \% \mathrm{HPD}=6.35-18.45)$. Comparative analyses of the tribe Delphinieae plastomes revealed that the loss of the rpl32 gene was shared by tribe Delphinieae, but the loss of the rps16 gene was lineage-specific (Fig. 1). The phylogenetic distribution indicated that the truncation of plastid-encoded matK had occurred independently in A. chiisanense and A. austrokoreense (Fig. 1). We found that all the sampled Delphinieae plastomes contained the plastid-encoded infA gene, which was previously annotated as a gene loss in some Aconitum species (Supplementary Fig. S4).

The transcriptome data of four Delphinieae species (Aconitum carmichaelii, C. orientalis, D. maackianum, and S. macrosperma) provide strong evidence for potential functional transfers of the rps 16 and rpl32 genes (Supplementary Table S2). The plastid-encoded rps16 has been functionally replaced by the nuclear-encoded mitochondrial rps16, whereas the plastid-encoded rpl32 has been subject to functional transfer to the nucleus (Fig. 1). In tribe Delphinieae, a gene substitution for the plastid $r p s 16$ was also identified because the $r p s 16$-like transcripts were not detected by a "blastn" search against the plastid-encoded rps 16 gene of $R$. macranthus; however, two different transcripts were detected using the query sequence of the nuclear-encoded plastid $r p s 16$ ( $M$. truncatula, AB365526). Phylogenetic analyses of the rps16 sequences from four Delphinieae species and the nuclear-encoded plastid and mitochondrial rps16 (AB365527) of M. truncatula indicated two different origins for the transcripts (Supplementary Fig. S5).

Interestingly, the A. carmichaelii transcriptome contains two divergent $r p l 32$ copies with $88.9 \%$ nucleotide sequence identity (Supplementary Fig. S6A), whereas Consolida, Delphinium, and Staphisagria each have only one transcript for $r p l 32$. All the predicted open reading frames (ORFs) have a transcript peptide with a conserved ribosomal L32 domain (Supplementary Table S2). To confirm the multiple versions and evaluate their intron-containing or intronless status, a nuclear copy was amplified from the A. pseudolaeve by specifically targeting the nuclear-encoded rpl32. RT-PCR analysis revealed that $A$. pseudolaeve also contains at least two different versions of $r$ pl32 with no premature stop codons or frameshift-producing insertions or deletions (indels) and that 


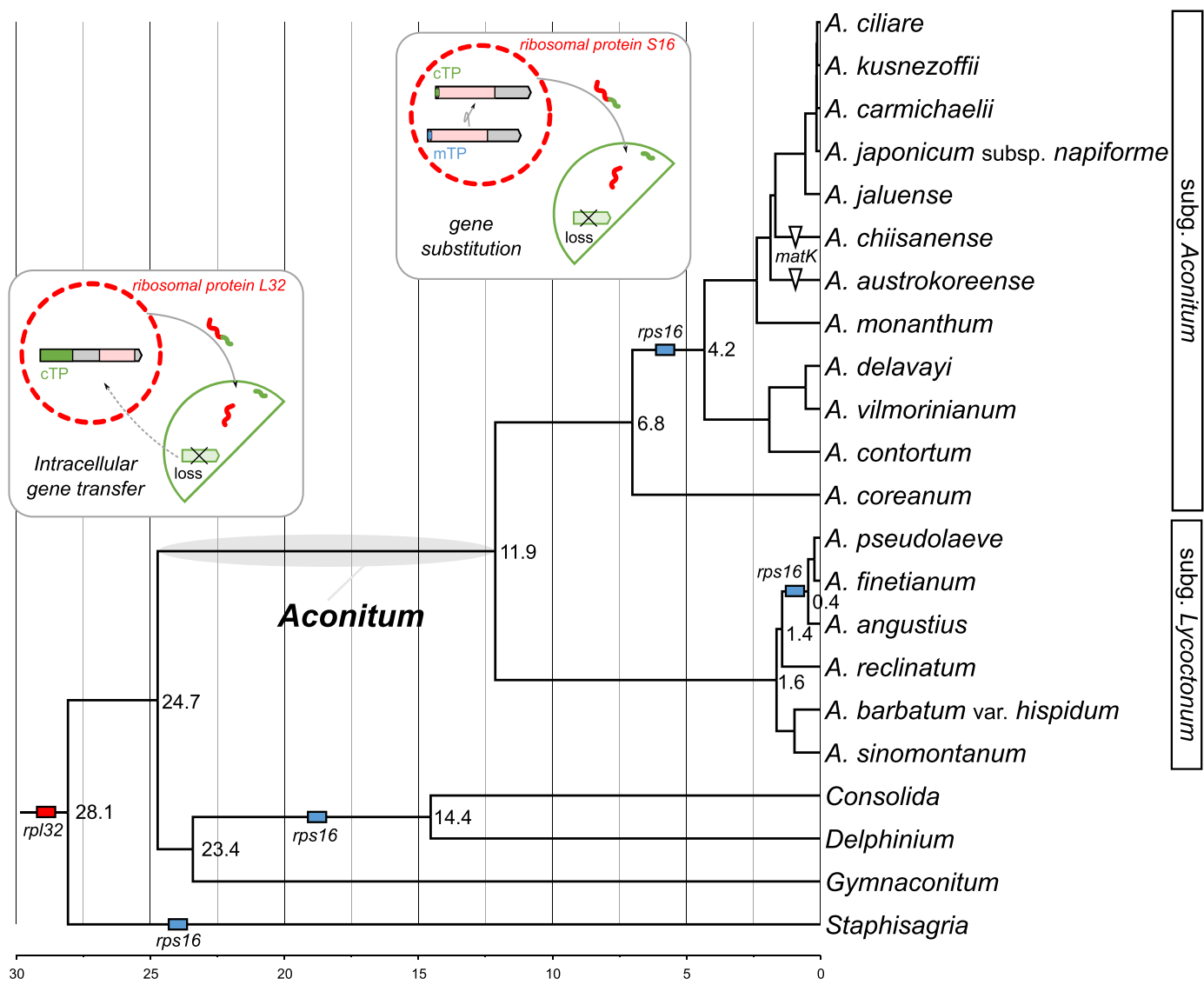

Figure 1. Phylogenetic distribution of gene content among tribe Delphinieae with divergence times. Numbers at nodes indicate divergence time estimates in Mya. Schematic diagram of functional transfers of the rpl32 and rps 16 genes from plastids to the nucleus by intracellular gene transfer and gene substitution, respectively. Pink boxes indicate the conserved domains of each gene. The green and blue boxes in the N-terminus indicate the chloroplast (cTP) and mitochondrial (mTP) transit peptides, respectively. The figures were constructed in BEAST2 v2.5.1 (https://www.beast2.org/) and InkScape v0.92.2 (https://inkscape.org).

\begin{tabular}{|l|l|l|l|l|l|l|l|}
\hline & $\begin{array}{l}\text { Intact } \\
\text { copy } \\
\text { number }\end{array}$ & Length & Align. & Identity & $\begin{array}{l}\text { GC } \\
\text { content }\end{array}$ & Type & $\begin{array}{l}\text { Gene } \\
\text { integrity }\end{array}$ \\
\hline A. austrokoreense & 6 & $429-438$ & 450 & $86.2-97.9$ & $52.2-53.8$ & Indels/SNPs & I \& P (1) \\
\hline A. barbatum & 6 & 444 & 444 & $99.1-99.8$ & $54.5-55.2$ & SNPs & I \\
\hline A. carmichaelii & 2 & 435,438 & 450 & 88.9 & $53.2,53.8$ & Indels & I \\
\hline A. ciliare & 3 & $408-429$ & 441 & $83.0-95.3$ & $51.7-52.2$ & Indels/SNPs & I \& P (3) \\
\hline A. coreanum & 5 & $435-441$ & 441 & $93-99.8$ & $50.1-52.2$ & Indels/SNPs & I \\
\hline A. glandulosum & 2 & 435,438 & 450 & 87.8 & $53.6,53.7$ & Indels & I \& P (3) \\
\hline A. japonicum & 5 & $408-441$ & 441 & $81.9-99.8$ & $51.7-53.1$ & Indels/SNPs & I \& P (2) \\
\hline A. kusnezoffi & 3 & $408-429$ & 441 & $82.5-96.3$ & $51.6-52.9$ & Indels/SNPs & I \& P (3) \\
\hline A. monanthum & 4 & $429-438$ & 450 & $88.4-99.8$ & $52.4-53.4$ & Indels/SNPs & I \& P (1) \\
\hline A. pseudolaeve & 2 & 435,438 & 441 & 95.5 & $54.6,55.6$ & Indels & I \\
\hline
\end{tabular}

Table 1. Nuclear-encoded rpl32 homologs in the genus Aconitum. I, intact gene; P, pseudogene. The number in parentheses indicates the copy number of pseudogene.

both copies are transcribed (Supplementary Fig. S6B). Alignment of the DNA and RNA nucleotide sequences revealed that the nuclear-encoded rpl32 is an intronless gene (Supplementary Fig. S6B).

Aconitum contains multiple versions of the nuclear-encoded plastid $r p l 32$ gene. Eight additional species of Aconitum representing two subgenera were surveyed for identification of the nuclear-encoded plastid rpl32 copy using PCR, cloning, and Sanger sequencing. We found that multiple copies were also present as intact genes or pseudogenes in most of the examined Aconitum species (Table 1 and Supplementary Fig. S6C-J). 


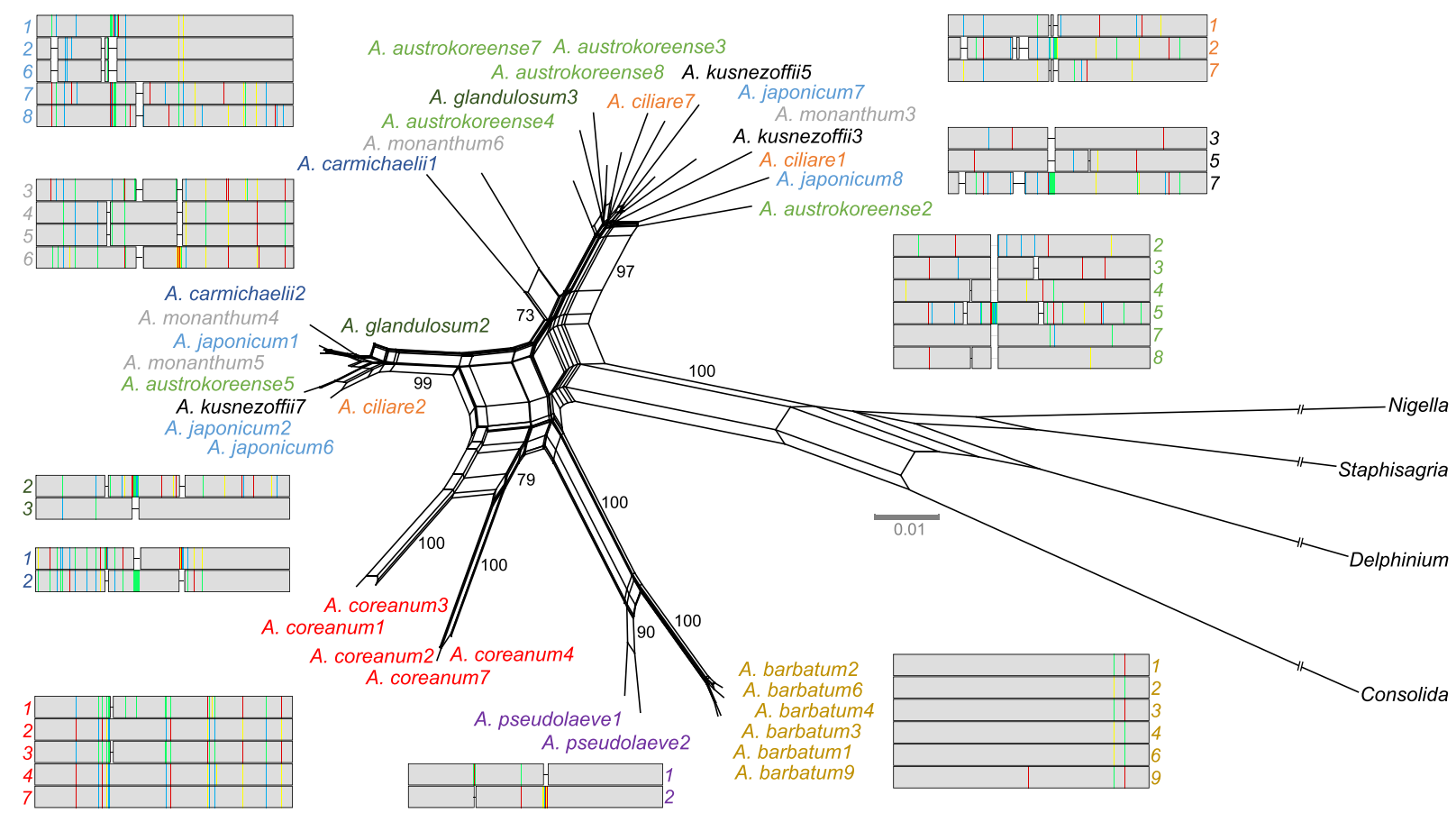

Figure 2. Phylogenetic network of nuclear-encoded plastid rpl32 homologs. The numbers after each species indicate the paralogs of $r$ pl32. The colored taxon name corresponds to the colored numbers after the alignment. The colored bars on the alignment map indicate nucleotide sites that differ from the consensus sequence (red, A; blue, C; yellow, G; green, T) (See Supplementary Fig. S6 for more details). The figures were constructed in SplitsTree v4.10 (https://en.bio-soft.net/tree/SplitsTree.html) and Geneious R7 v7.1.8 (https://www.geneious. com), and modified in InkScape v0.92.2 (https://inkscape.org).

Notably, all 10 examined Aconitum species contained at least two copies of the intact nuclear-encoded plastid rpl32, which ranged from $408 \mathrm{bp}$ to $441 \mathrm{bp}$ in length (Table 1). The nucleotide sequence alignments of the nuclear copies showed that they were highly divergent ( $88.5 \%$ nucleotide sequence identity), containing single nucleotide polymorphisms (SNPs) and indels (Table 1 and Fig. 2). No gene conversion between the paralogs of the nuclear-encoded $r p l 32$ gene was detected by GENECONV.

A Neighbor-Net analysis was used to show the clustering of the Aconitum rpl32 sequences (Fig. 2). The paralogous copies from A. coreanum, A. barbatum, and A. pseudolaeve were grouped together, respectively, but the others were scattered within two clusters. Phylogenetic analyses of the nuclear-encoded plastid $r p l 32$ homologs from tribe Delphinieae revealed five main lineages with high bootstrap support (Fig. 3A and Supplementary Fig. S7). We hereafter refer to them as alpha $(\alpha)$, beta $(\beta)$, gamma $(\gamma)$, delta $(\delta)$, and epsilon $(\varepsilon)$. The phylogenetic relationships of the nuclear rpl32 sequences were not consistent with the plastid phylogenomic relationships (Fig. $3 \mathrm{~B}$ and Supplementary Fig. S2). For example, the rpl32 gene tree showed that clade III (corresponding to lineage $\alpha$ ) was basal to the other clades. The next diverging clade was lineage $\beta$ (clade III), followed by lineage $\gamma$ (clade II). The subgenus Lycoctonum (clade I: lineage $\varepsilon$ ), which is an early-diverging lineage in Aconitum, was nested within clade II (lineage $\delta$ ). To examine rate variation in the duplicated genes, the nonsynonymous $\left(d_{\mathrm{N}}\right)$ and synonymous $\left(d_{\mathrm{S}}\right)$ substitution rates for their homologs were computed using the $r p l 32$ gene tree as a constraint tree. We discovered higher synonymous divergence in the homologs of lineage $\varepsilon$ than in those of the other lineages (Supplementary Fig. S8). The synonymous branch for lineage $\varepsilon$ was 3.0-8.6 times longer than those for the other lineages. Three branches with $d_{\mathrm{N}} / d_{\mathrm{S}}>1$ were detected in lineage $\beta$ (A. kusnezoffii3\&5 and A. japonicum 7 , Supplementary Fig. S8), but likelihood ratio tests (LRTs) showed that none of them were significantly different $(p=1.00$ after Bonferroni correction) (Supplementary Table S3). Taken together, the phylogenetic incongruence and the different substitution rates of the homologs indicated that each lineage had an ancestral rpl32 copy unique to that lineage and that the ancestral rpl32 copies were shared with a single ancestral lineage.

Thus, we propose a model for the evolution of the nuclear-encoded $r p l 32$ gene after IGT to account for the gene duplication (GD) events in the Aconitum lineage (Fig. 3B and Supplementary Fig. S7). In this model of the ancestral GD, the most parsimonious interpretation is that the nuclear $r p l 32$ gene first underwent multiple GD events (at least four times) and that five rpl32 paralogs existed in the common ancestor of Aconitum until speciation event that split the ancestor into two new lineages (Fig. 3B and Supplementary Fig. S7). Then, one of the new lineages split into two additional new lineages, followed by stochastic loss. After the ancestral GD, the gene tree showed recurrent lineage-specific duplication and speciation events, and the patterns of the recent GD events were more complicated (Supplementary Fig. S7). The histograms of the synonymous substitution rates for the homologous pairs supported two potential major GD events (Fig. 3C). We observed one and two peaks in the recent and ancestral GDs, respectively. Four peaks in the ancestral GD were detected by the adjusted Gaussian (Fig. 3C), supporting the model. The duplicated copies derived from the ancestral GD event exhibited greater 

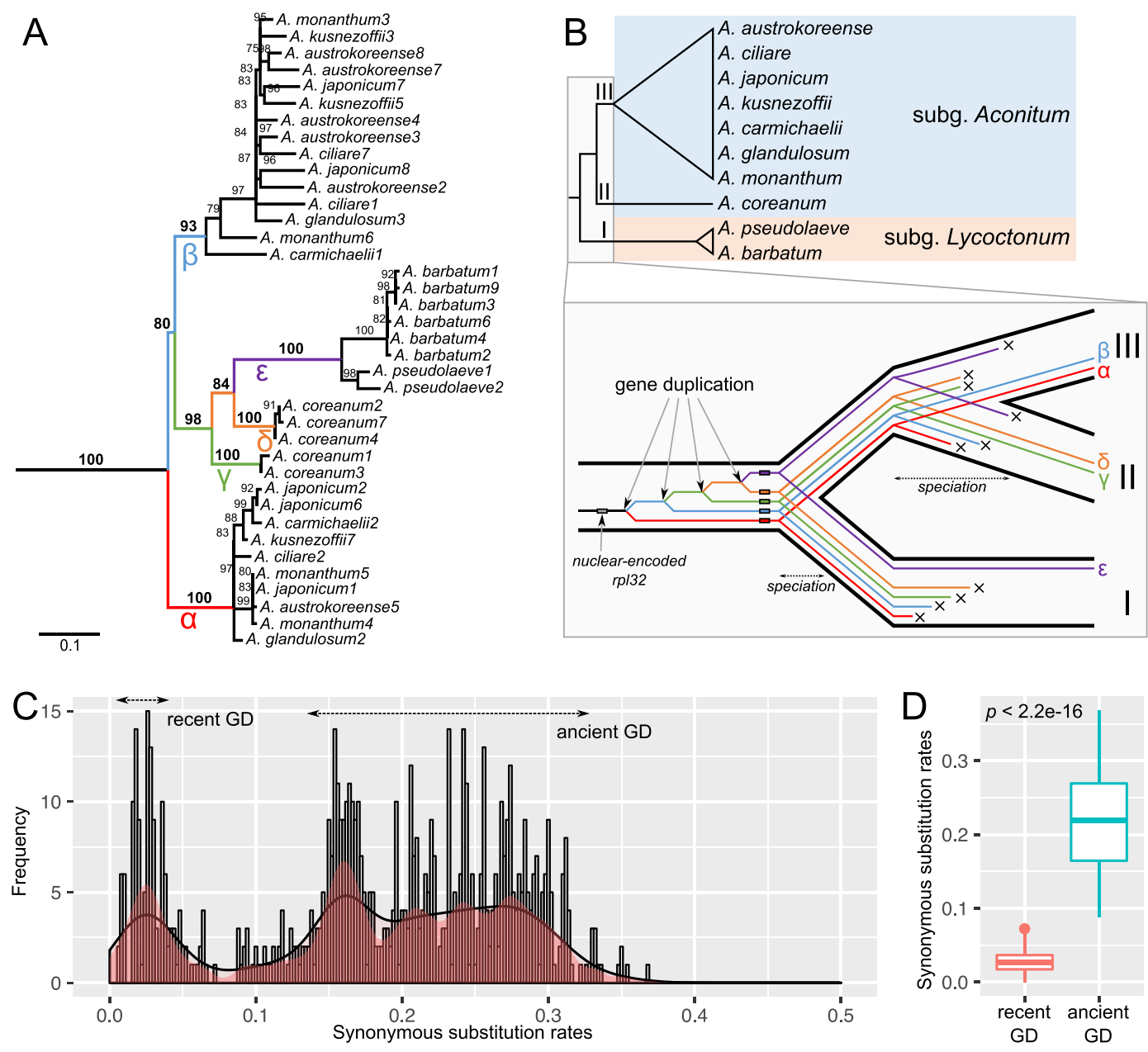

Figure 3. Nuclear-encoded plastid rpl32 gene duplication events. (A) ML gene tree based on the nuclearencoded $r p l 32$ nucleotide sequences. The numbers after each species indicate the paralogs of $r p l 32$. Bootstrap support values $>70 \%$ are shown on the branches. (B) Phylogenetic relationships within Aconitum. Ancestral gene duplications are first followed by a speciation and then by asymmetric gene losses. Three lineages have one or two ancestral paralogs. (C) Histograms of the $d_{\mathrm{S}}$ distribution of the nuclear-encoded $r p l 32$ homologs derived from recent and ancient GD events. The $d_{\mathrm{S}}$ distribution was fitted using Gaussian mixture models. The $d_{\mathrm{S}}$ peaks corresponding to each GD. (D) Comparison of sequence divergence between homologs from two different GD events. The figures were constructed in IQ-TREE v1.6.2 (https://iqtree.org), R v3.4.2 (https://r-project.org) and InkScape v0.92.2 (https://inkscape.org).

divergence than those from the recent GD event (Wilcoxon rank-sum test, $p<0.01$ ) (Fig. 3D). The divergence time estimates suggested that the ancestral GD events occurred between 11.9 and 24.7 Mya and that the recent GD events occurred $\leq 4.2$ Mya (Fig. 1 ).

Furthermore, we estimated the $d_{\mathrm{N}}$ and $d_{\mathrm{S}}$ substitution rates for the plastid-encoded gene and compared them to address whether the nucleotide substitution rates for the plastid-encoded ribosomal protein large ( $r p l$ ) subunits in Aconitum were elevated by the presence of multiple versions of the nuclear-encoded $r p l 32$. Only the $d_{\mathrm{N}}$ values for the plastid $r p l$ subunits that assemble the nuclear-encoded $r p l 32$ protein were significantly higher in Aconitum than in other genera (Wilcoxon rank-sum test, $p<0.01$; Fig. 4 and Supplementary Fig. S8).

Heteroplasmic variation in the plastid-encoded maturase K. The two published Aconitum plastomes, those of $A$. austrokoreense and A. chiisanense, harbored a truncated plastid maturase $\mathrm{K}(\mathrm{matK})$ gene in their genomes. Comparisons of the $m a t K$ copies from these species with those of related species revealed that former shared a single-base (T) deletion (position 946) that generated a premature stop codon (Fig. 5A). Investigation of the conserved domain revealed that the mat $K$ copies had approximately half of the gene, which contained only the conserved "matK_TrnK amino terminal region" domain and lacked the "Type II intron maturase" domain (Fig. 5A). To confirm this variation, we examined the polymorphisms in the plastid-encoded matK by cloning and Sanger sequencing an additional five individuals of $A$. austrokoreense (Fig. 5B). High polymorphic variation was detected in the homopolymeric (T) region of the 25 isolates of the five A. austrokoreense individuals; this region ranged in length from 8 to $11 \mathrm{bp}$ (Fig. 5B). Two nontriplet deletions caused frameshifts, but a triplet "TTT" deletion resulted in a single amino acid loss (phenylalanine). 


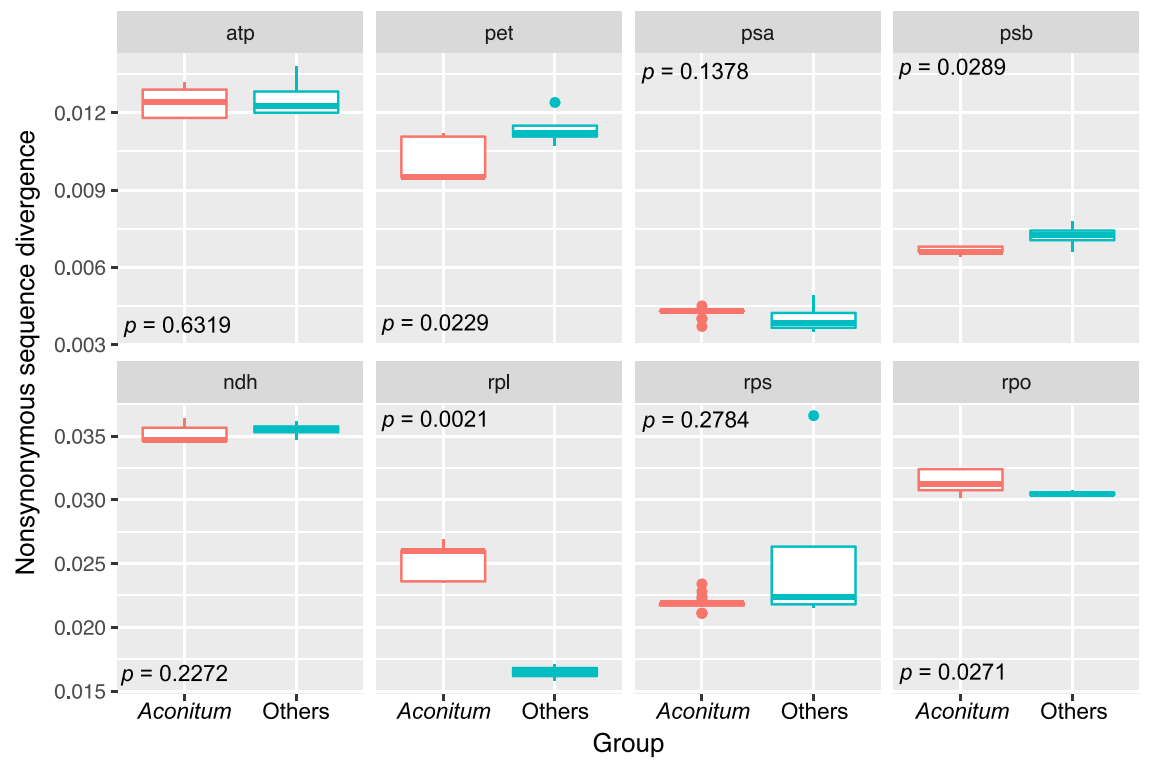

Figure 4. Box plots of $d_{\mathrm{N}}$ values for plastid functional gene groups in Aconitum and other genera. The box represents values between quartiles, the solid lines extend to the minimum and maximum values, and the horizontal lines in the boxes show the median values. Aconitum, red; other genera, blue.

\section{Discussion}

We have shown that all analyzed Aconitum plus Consolida, Delphinium, Gymnaconitum, and Staphisagria species lack the plastid-encoded rpl32 gene in their plastomes, suggesting an ancient loss in the ancestor of tribe Delphinieae. In contrast, the phylogenetic distribution of the sampled plastomes indicated that the plastid-encoded rps16 gene has been lost several times within the tribe. The transcriptomes indicated that the two plastid genes, rpl32 and rps16, were lost after their functional replacement by IGT and gene substitution in the nucleus, respectively. These evolutionary processes suggest that the inactivation of two plastid genes is favored after nuclear gene activation. It is well known that polyploidization and hybridization contribute to Aconitum species diversity ${ }^{18-20}$. Our findings raise fundamental questions about the fates of transferred genes in a polyploid plant. If IGT events occurred before polyploid formation, gene doubling would occur via polyploidization. If multiple organelle-targeted copies are present, what are the potential effects of these multiple nuclear copies on the nucleotide substitution rates of the subunits encoded in the plastids?

Surprisingly, we discovered that all the sampled Aconitum species contained multiple versions of the nuclear-encoded plastid rpl32. We observed that the rpl32 paralogs from each Aconitum species were highly divergent, having both SNPs and indels. However, the rpl32 phylogeny showed sporadic distribution and phylogenetic incongruence (Fig. 3A). These phenomena generally indicate that independent events took place in each lineage or that stochastic losses with vertical transfer happened after GD events. An alternative explanation for this phenomenon is horizontal gene transfer within the genus. The clustering of $r p l 32$ homologs and the $d_{\mathrm{S}}$ distribution signals clearly demonstrated that the multiple copies originated from duplications (ancient GD), which may be associated with WGD. The presence of $r p l 32$ pseudogenes in several Aconitum species suggested that their sporadic distribution is due to inactivation and eventual loss over time. Given the WGD signal and chromosome number, the genus Aconitum may have undergone multiple events of polyploidization followed by diploidization in the ancient lineage. Many diploid Aconitum $(2 \mathrm{n}=16)$ species contain two or more rpl32 paralogs, supporting the hypothesis of Aconitum paleopolyploidy. In contrast, it is unclear whether the recent GD is the result of WGD or smaller scale segmental or single-gene duplication. The recurrent GD and losses make it difficult to determine the mechanisms. The nuclear-encoded rps16 could also have been affected by a recent genome doubling event, but we did not find evidence for GD of $r p s 16$. This finding supports the hypothesis that recent GD events may involve multiple evolutionary factors rather than only WGD. However, it is possible that the duplicated $r p s 16$ genes were lost ancestrally, given their redundancy. Transcriptome data from other species are required to obtain a full understanding of the evolutionary history of WGD among the genus Aconitum. Duplicated plastid-targeted genes are reduced to a single-copy gene $e^{30}$, and reciprocal gene loss occurs between duplicated genes, which lead to divergence ${ }^{31}$. The nuclear rpl32 sequences found in some Aconitum species represent pseudogenes (Supplementary Fig. S6). Thus, the nuclear-encoded plastid rpl32 paralogs are expected to be retained as single-copy genes over time.

Highly divergent mutations in the nuclear-encoded genes that target organelles can drive compensatory changes in associated organelle genes ${ }^{32-34}$. In plants, cytonuclear coevolution has been observed in plastid protein complexes that include subunits encoded in the nuclear and plastid genomes ${ }^{35,36}$. After protein synthesis of the nuclear-encoded plastid rpl32 in the cytosol, they must be imported into the plastids and assembled into plastid-localized multisubunit complexes (i.e., with the products of $r p l 2, r p l 14, r p l 16, r p l 20, r p l 22, r p l 23, r p l 33$, and $r p$ l36). We tested the effects of the nuclear rpl32 copies on substitution rate changes in the plastid-encoded $r p l$ subunits. The plastid-encoded $r p l$ subunits of the genus Aconitum had highly accelerated nonsynonymous 
A

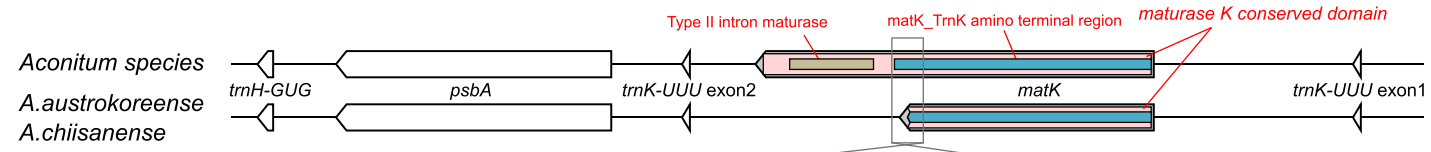
A.chiisanense

A. ciliare
A. kusnezoffii
A. japonicum subs. napiforme
A. carmichaelii
A. jaluense
A. chiisanense
A. austrokoreense
A. monanthum

B

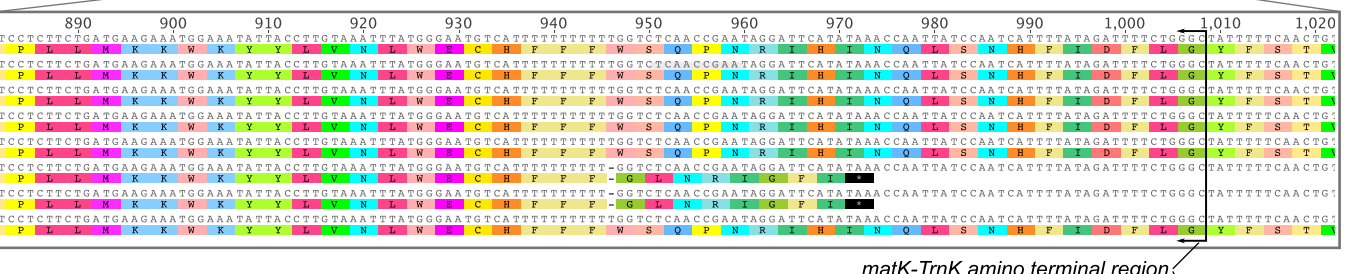

A. austrokoreense1 1 A. austrokoreense 1 A. austrokoreense 1 A. austrokoreense1_4 A. austrokoreense1 5 A. austrokoreense2 1 A. austrokoreense2 A. austrokoreense2_3 A. austrokoreense2 4

A. austrokoreense2 5 A. austrokoreense 31 A. austrokoreense3_2 A. austrokoreense 3 A. austrokoreense 3

A. austrokoreense 3

A. austrokoreense 4

A. austrokoreense4_2 A. austrokoreense4

A. austrokoreense4 4

A. austrokoreense 4

A. austrokoreense 5

A. austrokoreense5_2

A. austrokoreense 5

A. austrokoreense 5

A. austrokoreense 5

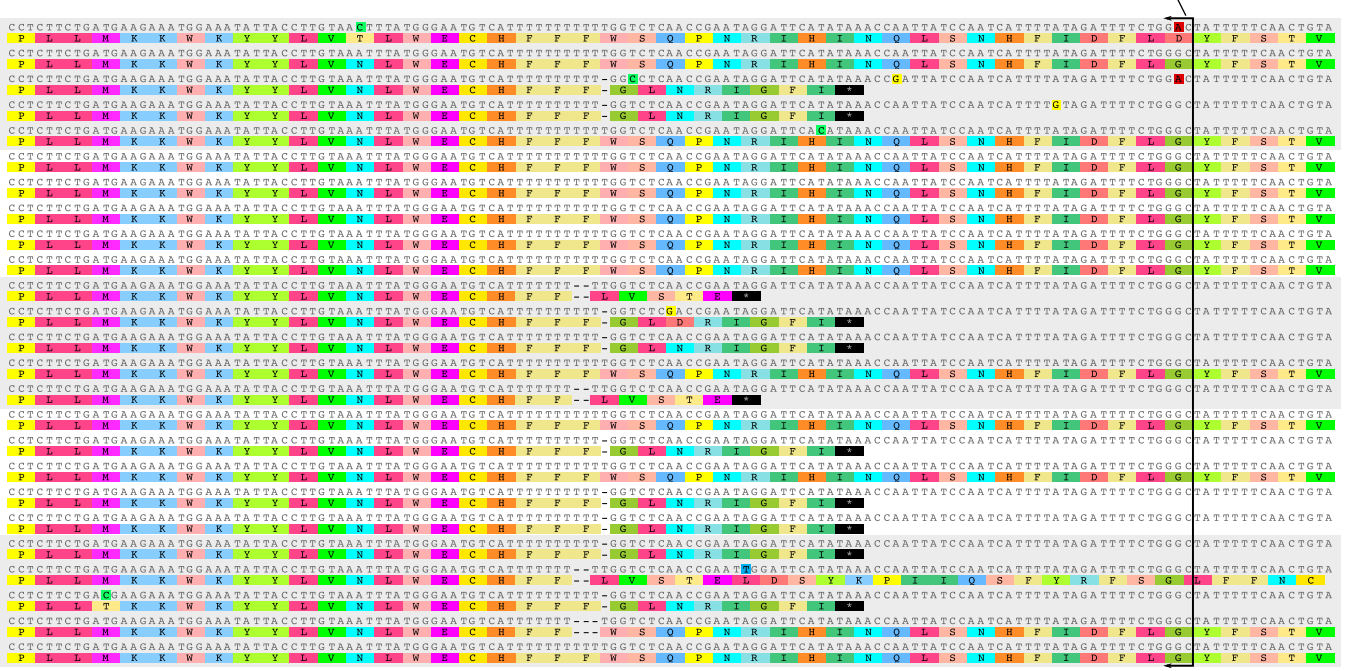

Figure 5. Characterization of the plastid-encoded matK genes of Aconitum. (A) Schematic diagram of the genetic region surrounding $m a t K$ in $A$. austrokoreense and A. chiisanense compared with other Aconitum species. Boxes inside the matK gene indicate the conserved domains (matK_TrnK amino terminal region: blue, Type II intron maturase: brown). Nucleotide sequence alignment of the matK hotspot region in eight Aconitum species. (B) Nucleotide sequences of the matK copies from five A. austrokoreense individuals. The solid lines (black) indicate the end of the conserved domain of the matK_TrnK amino terminal region.

substitution rates relative to the corresponding rates of the other analyzed genera (Fig. 4). These results lead us to conclude that highly divergent multiple copies of the nuclear-encoded $r p l 32$ may trigger compensatory changes in the eight plastid-encoded $r p l$ subunits. However, alternative mechanisms, such as relaxation of functional constraints $^{37}$ and cytonuclear incompatibilities ${ }^{36,38}$, can contribute to accelerated sequence evolution in the Aconitum plastid-encoded $r p l$ subunits. Further studies that include organelle ribosomes as well as nuclear-encoded regulatory factors are needed.

Maturase $\mathrm{K}(m a t K)$ is encoded within the intron of $\mathrm{tRNA}^{\mathrm{Lys}}(\operatorname{trnK}-U U U)$ in the plastids ${ }^{39}$. The matK protein is essential for the splicing of group II introns ${ }^{40}$, which target seven $c i s$-spliced introns from atpF, $r p l 2, r p s 12$, tRNA $^{\mathrm{Val}}(\operatorname{trn} V-U A C), \mathrm{tRNA}^{\text {Ile }}(\operatorname{trnI}-G A U), \mathrm{tRNA}^{\text {Ala }}(\operatorname{trn} A-U G C)$, and $\operatorname{trn} K-U U U^{41}$. Truncated matK genes have been documented in two Aconitum species ${ }^{29}$ that both lack the $\mathrm{C}$-terminal portion. Our analyses revealed that the matK genes of these two species lacked the conserved Type II intron maturase domain at the C-terminal (Fig. 5), suggesting a loss of functionality. To maintain its function in the plastids, mat $K$ would need to be functionally replaced by the nucleus. However, previous studies have suggested the parallel loss of all introns and plastid $m a t K^{42-44}$. The two Aconitum plastomes retain the seven target introns, indicating the necessity for matK in their plastids. Interestingly, PCR cloning and Sanger sequencing revealed heteroplasmic variation in the mat $K$ sequences of five A. austrokoreense individuals (Fig. 5). The presence of a triplet nucleotide deletion or no mutation at the same position indicated that they probably encode functional proteins in the A. austrokoreense plastid. These sequences that suggested the functionality of $m a t K$ are from the plastome and indicate that $A$. austrokoreense contains at least two versions in its plastome. High coverage sequencing of the A. austrokoreense plastome ${ }^{29}$ suggests that the truncated $m a t K$ copies exist predominantly in its plastome. However, we cannot exclude the possibility that some sequences were amplified from a nuclear plastid DNA (NUPT) or a mitochondrial plastid DNA (MIPT). Complete mitochondrial and nuclear genomes (including transcriptome) sequences are needed to better address this phenomenon. Heteroplasmic mutations are identified either within a cell or among cells; they can occur due to replication slippage, recombination, cytonuclear interaction, or diverse environmental factors $^{45-48}$. The frameshift mutation in the matK genes from the two Aconitum plastomes may be associated with chlorophyll deficiency ${ }^{49}$. 


\section{Conclusions}

This study provides valuable insights into the patterns of genome evolution across tribe Delphinieae. In particular, duplication of the nuclear-encoded rpl32 after ITG was found in all the sampled Aconitum species of tribe Delphinieae. Our phylogenetic analysis showed five diverse clades of $r p l 32$ homologs, implying that at least five paralogs existed in the common ancestor of the genus Aconitum. The $d_{\mathrm{S}}$ distribution signal suggested that additional rpl32 paralogs have arisen via duplication in recent Aconitum evolution. The ancient GD appears more likely to have been caused by WGD than by a single event, whereas the recent GDs have involved multiple processes, such as WGD, single-gene duplication, hybridization, or horizontal gene transfer. Rate analyses supported the hypothesis that selection for compensatory changes in response to multiple divergent $r p l 32$ paralogs contributes to an elevated rate of evolution in plastid $r p l$ subunits. In addition, heteroplasmy in the matK within and among individuals is identified, but the evolutionary forces driving this phenomenon is nuclear. To obtain a comprehensive understanding of the plastid heteroplasmy among Aconitum genomes, multiple genome sequences from a population are required.

\section{Methods and Methods}

Genome sequencing, assembly and annotation. Total genomic DNA (gDNA) of Aconitum pseudolaeve, Consolida orientalis, Delphinium maackianum, Staphisagria macrosperma, and Nigella damascena was isolated from fresh leaf tissues using the DNeasy Plant Mini Kit (Qiagen, Hilden, Germany) following the manufacturer's protocol (Supplementary Table S4). The five gDNAs were sequenced using the Illumina HiSeq 2500 sequencing platform (Illumina, San Diego, CA), generating approximately $6 \mathrm{~Gb}$ of paired-end (PE) reads from a 550-bp insert library.

The PE reads were assembled de novo with Velvet v1.2.10 ${ }^{50}$ using multiple $k$-mers (69 to 91 ) on a 32 -core $3.33 \mathrm{GHz}$ Linux work station with $512 \mathrm{~GB}$ of memory. Five plastomes were determined following the methods in Park et al. ${ }^{51}$. We annotated the plastomes using a BLAST-like algorithm in Geneious R7 v7.1.8 (www. geneious.com) ${ }^{52}$ with the genes of the model plant tobacco (Nicotiana tabacum, NC_001879) as the reference and confirmed the open reading frames (ORFs). All tRNA genes were predicted using tRNAscan-SE v2.0.3 ${ }^{53}$ and ARAGORN v1.2.38 ${ }^{54}$. The plastomes were deposited in GenBank (accession number MN648400-MN648404). Circular plastome maps were drawn with OGDRAW v1.3.1 (chlorobox.mpimp-golm.mpg.de/OGDraw.html ${ }^{55}$.

Phylogeny and divergence times. A single alignment data set was concatenated with 77 protein-coding gene alignments from 22 species of tribe Delphinieae and two outgroups, $N$. damascena and Ranunculus macranthus (Supplementary Table S5). The individual gene alignments were generated based on the back-translation approach with MAFFT v7.01756 in Geneious R7. The maximum likelihood (ML) tree was inferred from the concatenated data set under each of 17 partitioning schemes (Supplementary Table S6) using IQ-TREE v1.6.2 ${ }^{57}$ with the ultrafast bootstrap algorithm ${ }^{58}$ (1,000 replicates). Divergence times were inferred in BEAST2 v2.5.1 ${ }^{59}$ using a concatenated alignment data set that excluded the two outgroups. A calibration point estimated for tribe Delphinieae $^{60}($ mean $=28.95, \mathrm{SD}=5$, range 20.7-37.2 Ma) was used with a normal prior distribution as the root age constraint.

Identification of functional transfer to the nucleus. Total RNA was isolated from fresh leaves of $C$. orientalis, D. maackianum, and S. macrosperma using the methods of Ghawana et al. ${ }^{61}$ and treated with DNase I (Invitrogen, USA). The RNAs were sequenced using the Illumina HiSeq 2500 sequencing platform, generating approximately $6 \mathrm{~Gb}$ of PE reads. To evaluate potential IGT sources, transcriptomes from C. orientalis, D. maackianum, and S. macrosperma were assembled de novo with Trinity v2.5.162. In addition, Aconitum carmichaelii (SRR6225422) and Nigella sativa (SRR341997) were included. Nuclear-encoded protein genes (rpl32 and rps16) for plastids were identified by "blastn" searches ( $e$-value cutoff of 1e-10) using BLAST $+v 2.7 .1^{63}$, employing plastid-encoded rpl32 and rps 16 from $R$. macranthus as the query sequences. We also used the Medicago truncatula (AB365526) nuclear-encoded plastid rps16, which was supplanted by the nuclear-encoded mitochondrial rps $16^{28}$. The chloroplast transit peptide (cTP) and its cleavage site were predicted by TargetP v1.1 ${ }^{64}$. The NCBI Conserved Domain Database (CDD) was used for functional domain annotation ${ }^{65}$.

Survey of variability in the nuclear-encoded plastid $r$ pl32. To better understand the evolutionary fate of the nuclear-encoded plastid rpl32, eight Aconitum species were sampled (Supplementary Table S7). Total genomic DNA was isolated from herbarium specimens using the methods of Allen et al. ${ }^{66}$. To detect the nuclear rpl32 copies, polymerase chain reaction (PCR) was carried out using the total genomic DNA. Primer pairs were designed based on sequences of the nuclear rpl32 copies from A. carmichaelii using Primer $3^{67}$ in Geneious R7 (forward: CCATGGCSACATCTCTACTACCMA and reverse: CACCACAAAGTAGATGGGGCTTGG). The PCR was $50 \mu \mathrm{l}$ in volume, including $38.75 \mu \mathrm{l}$ of distilled water, $5 \mu \mathrm{l}$ of $10 \times$ Taq Reaction Buffer, $1 \mu \mathrm{l}$ of $10 \mathrm{mM}$ dNTPs, $0.25 \mu$ l of DiaStar Taq polymerase ( 5 units/ $\mu$ l, Solgent Co., Daejeon, South Korea), $1 \mu$ l of each primer $(10 \mathrm{pmol} / \mu \mathrm{l})$, and $1 \mu \mathrm{l}$ of total genomic DNA $(20 \mathrm{ng})$. All reactions included an initial denaturation step $\left(95^{\circ} \mathrm{C}\right.$ for $2 \mathrm{~min}), 35$ cycles of denaturation $\left(95^{\circ} \mathrm{C}\right.$ for $\left.20 \mathrm{~s}\right)$, annealing $\left(60^{\circ} \mathrm{C}\right.$ for $\left.40 \mathrm{~s}\right)$, extension $\left(72^{\circ} \mathrm{C}\right.$ for $\left.1 \mathrm{~min}\right)$ and final extension $\left(72^{\circ} \mathrm{C}\right.$ for $5 \mathrm{~min}$ ). The PCR products were purified using a PCR purification kit (MGmed, South Korea) following the manufacturer's protocol. First, we sequenced a product from A. pseudolaeve using an ABI 3730xl DNA Analyzer (Applied Biosystems, California, USA) at Solgent Co. The results showed a pattern of mixed nonspecific PCR products. Thus, the purified products from all exampled Aconitum species were cloned using the T-Blunt PCR Cloning Kit (Solgent Co., Daejeon, South Korea) following the manufacturer's protocol. White colonies were picked, and direct colony PCRs were performed using Solg $h$-Taq DNA polymerase (Solgent Co., Daejeon, South Korea) with the M13 (-20) forward/reverse primers. The nucleotide sequences of isolated clones were determined by an automated DNA sequencer. The data set was aligned at the protein level by MAFFT 
using the translation-align function in Geneious R7. Neighbor-Net splits graphs of the nuclear-encoded rpl32 data were constructed using SplitsTree $\mathrm{v} 4.10^{68}$ with uncorrected $p$ distance ${ }^{69}$. The gene tree was inferred from the nuclear-encoded rpl32 paralog data set using IQ-TREE with the ultrafast bootstrap algorithm (1,000 replicates). Gene duplication events were inferred using Zmasek and Eddy's algorithm ${ }^{70}$ in MEGA X ${ }^{71}$.

To confirm that the two nuclear rpl32 copies were transcribed, total RNA isolation for A. pseudolaeve was performed as described above. Reverse transcription (RT)-PCR was performed using ImProm-II Reverse Transcriptase (Promega, USA) with random hexamers. PCR amplification was carried out with primer pairs specific to the nuclear-encoded $r$ pl32. PCR purification and sequencing were performed as described above.

Estimation of sequence divergence. Nonsynonymous and synonymous substitution rates were calculated in PAML v4. $8^{72}$ using the ML tree as a constraint tree. Analyses were performed using the CODEML program, employing the F3 44 codon frequency model, and gapped regions were excluded with the "cleandata $=1$ " option. The estimations of rate variation were performed on the concatenated gene sets for the functional groups ATP synthase (atp), cytochrome b6f (pet), NADH-plastoquinone oxidoreductase ( $n d h)$, photosystem I $(p s a)$, photosystem II ( $p s b)$, RNA polymerase $(r p o)$, and ribosomal protein small $(r p s)$ and large ( $r p l)$ subunits. Likelihood ratio tests (LRTs) were performed to test the $d_{\mathrm{N}} / d_{\mathrm{S}}$ changes. The null model was fixed across the entire tree, whereas an alternative model allowed different values of $d_{\mathrm{N}} / d_{\mathrm{S}}$ for branches in the phylogenetic tree.

To test for evidence of selection acting on duplicated genes, the $d_{\mathrm{N}}$ and $d_{\mathrm{S}}$ values for the nuclear-encoded $r p l 32$ were calculated. We also estimated the $d_{\mathrm{S}}$ values between the pairs of $r$ pl32 homologs, and Gaussian mixture models were fitted using R v3.4.2 $2^{73}$ to identify significant peaks. Statistical analyses were conducted with R, and the Bonferroni correction for multiple comparisons was applied.

Homopolymer length variation in the plastid maturase $\mathrm{K}$ gene. A truncated form of the plastid maturase K (matK) was identified in two published (A. austrokoreense (NC_031410) and A. chiisanense (NC_029829)) plastomes from tribe Delphinieae. A primer set was designed to amplify a product around the homopolymer site for A. austrokoreense (matKF: GGTTCAAATCCTTCGTTGTTGGAT and matKR: ATATCAGAATCGGATGAATCGGCC). Five gDNAs of $A$. austrokoreense were isolated from herbarium specimens (Supplementary Table S7). PCRs were performed as described above using Solg Pfu DNA polymerase (Solgent Co., Daejeon, South Korea) to reduce the error rate at homopolymers. PCR amplification, purification, cloning and sequencing were performed as described above.

The CDD was also used to detect conserved domains. The nucleotide sequences of the plastid matK copies were aligned with MUSCLE ${ }^{74}$ in Geneious R7.

Received: 4 December 2019; Accepted: 30 January 2020;

Published online: 17 February 2020

\section{References}

1. Ohno, S. Evolution by gene duplication. (Springer-Verlag, 1970).

2. Panchy, N., Lehti-Shiu, M. \& Shiu, S.-H. Evolution of gene duplication in plants. Plant Physiol. 171, 2294-2316, https://doi. org/10.1104/pp.16.00523 (2016).

3. Lynch, M. \& Conery, J. S. The evolutionary fate and consequences of duplicate genes. Science 290, 1151-1155, https://doi. org/10.1126/science.290.5494.1151 (2000).

4. Force, A. et al. Preservation of duplicate genes by complementary, degenerative mutations. Genet 151, 1531-1545 (1999).

5. Qian, W., Liao, B. Y., Chang, A. Y. \& Zhang, J. Maintenance of duplicate genes and their functional redundancy by reduced expression. Trends Genet. 26, 425-430, https://doi.org/10.1016/j.tig.2010.07.002 (2010).

6. Soltis, D. E., Soltis, P. S. \& Tate, J. A. Advances in the study of polyploidy since Plant speciation. New Phytol. 161, 173-191, https:// doi.org/10.1046/j.1469-8137.2003.00948.x (2004).

7. Wendel, J. F. Genome evolution in polyploids. Plant Mol. Biol. 42, 225-249 (2000).

8. Soltis, P. S. \& Soltis, D. E. The role of hybridization in plant speciation. Annu. Rev. Plant. Biol. 60, 561-588, https://doi.org/10.1146/ annurev.arplant.043008.092039 (2009).

9. Jiao, Y. et al. Ancestral polyploidy in seed plants and angiosperms. Nature 473, 97-100, https://doi.org/10.1038/nature09916 (2011).

10. Soltis, D. E. et al. Polyploidy and angiosperm diversification. Am. J. Bot. 96, 336-348, https://doi.org/10.3732/ajb.0800079 (2009).

11. Otto, S. P. The evolutionary consequences of polyploidy. Cell 131, 452-462, https://doi.org/10.1016/j.cell.2007.10.022 (2007).

12. Mandáková, T., Joly, S., Krzywinski, M., Mummenhoff, K. \& Lysak, M. A. Fast diploidization in close mesopolyploid relatives of Arabidopsis. Plant Cell 22, 2277-2290, https://doi.org/10.1105/tpc.110.074526 (2010).

13. Pontes, O. et al. Chromosomal locus rearrangements are a rapid response to formation of the allotetraploid Arabidopsis suecica genome. Proc. Natl Acad. Sci. U.S.A. 101, 18240-18245, https://doi.org/10.1073/pnas.0407258102 (2004).

14. Madlung, A. et al. Genomic changes in synthetic Arabidopsis polyploids. Plant J. 41, 221-230, https://doi.org/10.1111/j.1365313X.2004.02297.x (2005).

15. Tamura, M. In Die natürlichen Pflanzenfamilien Vol. 17aIV (ed. Hiepko, P.) (Ducker \& Humboldt, Berlin., 1995).

16. Jabbour, F., Ronse De Craene, L. P., Nadot, S. \& Damerval, C. Establishment of zygomorphy on an ontogenic spiral and evolution of perianth in the tribe Delphinieae (Ranunculaceae). Ann. Bot 104, 809-822, https://doi.org/10.1093/aob/mcp162 (2009).

17. Jabbour, F. \& Renner, S. S. Spurs in a Spur: Perianth Evolution in the Delphinieae (Ranunculaceae). Int. J. Plant. Sci. 173, 1036-1054, https://doi.org/10.1086/667613 (2012).

18. Kong, H., Zhang, Y., Hong, Y. \& Barker, M. S. Multilocus phylogenetic reconstruction informing polyploid relationships of Aconitum subgenus Lycoctonum (Ranunculaceae) in China. Plant Syst. Evol. 303, 727-744, https://doi.org/10.1007/s00606-017-1406-y (2017).

19. BlanchÈ, C., Bosch Daniel, M., LÛpez-Pujol, J. \& Simon, J. DCDB: an updated online database of chromosome numbers of tribe Delphinieae (Ranunculaceae). (2016).

20. Sutkowska, A., Boroń, P., Warzecha, T., Dębowski, J. \& Mitka, J. Hybridization and introgression among three Aconitum (Ranunculaceae) species of different ploidy levels in the Tatra Mountains (Western Carpathians). Plant Spec. Biol. 32, 292-303, https://doi.org/10.1111/1442-1984.12162 (2017).

21. Park, I. et al. The complete chloroplast genome sequence of Aconitum coreanum and Aconitum carmichaelii and comparative analysis with other Aconitum species. PLoS One 12, e0184257-e0184257, https://doi.org/10.1371/journal.pone.0184257 (2017).

22. Kong, H., Liu, W., Yao, G. \& Gong, W. A comparison of chloroplast genome sequences in Aconitum (Ranunculaceae): a traditional herbal medicinal genus. PeerJ 5, e4018-e4018, https://doi.org/10.7717/peerj.4018 (2017). 
23. Meng, J. et al. Comparative analysis of the complete chloroplast genomes of four Aconitum medicinal species. Molecules 23, https:// doi.org/10.3390/molecules23051015 (2018).

24. Timmis, J. N., Ayliffe, M. A., Huang, C. Y. \& Martin, W. Endosymbiotic gene transfer: organelle genomes forge eukaryotic chromosomes. Nat. Rev. Genet. 5, 123-135, https://doi.org/10.1038/nrg1271 (2004).

25. Cusack, B. P. \& Wolfe, K. H. When gene marriages don't work out: divorce by subfunctionalization. Trends Genet. 23, 270-272, https://doi.org/10.1016/j.tig.2007.03.010 (2007).

26. Park, S., Jansen, R. K. \& Park, S. Complete plastome sequence of Thalictrum coreanum (Ranunculaceae) and transfer of the rpl32 gene to the nucleus in the ancestor of the subfamily Thalictroideae. BMC Plant Biol. 15, 40, https://doi.org/10.1186/s12870-0150432-6 (2015).

27. Ueda, M. et al. Loss of the rpl32 gene from the chloroplast genome and subsequent acquisition of a preexisting transit peptide within the nuclear gene in Populus. Gene 402, 51-56, https://doi.org/10.1016/j.gene.2007.07.019 (2007).

28. Ueda, M. et al. Substitution of the gene for chloroplast RPS16 was assisted by generation of a dual targeting signal. Mol. Biol. Evol. 25, 1566-1575, https://doi.org/10.1093/molbev/msn102 (2008).

29. Choi, J.-E., Kim, G.-B., Lim, C.-E., Yu, H.-J. \& Mun, J.-H. The complete chloroplast genome of Aconitum austrokoreense Koidz. (Ranunculaceae), an endangered endemic species in Korea. Mitochondrial DNA Part. B 1, 688-689, https://doi.org/10.1080/238023 59.2016.1219644 (2016)

30. De Smet, R. et al. Convergent gene loss following gene and genome duplications creates single-copy families in flowering plants. Proc. Natl Acad. Sci. U.S.A. 110, 2898-2903, https://doi.org/10.1073/pnas.1300127110 (2013).

31. Scannell, D. R., Byrne, K. P., Gordon, J. L., Wong, S. \& Wolfe, K. H. Multiple rounds of speciation associated with reciprocal gene loss in polyploid yeasts. Nature 440, 341-345, https://doi.org/10.1038/nature04562 (2006).

32. Havird, J. C., Whitehill, N. S., Snow, C. D. \& Sloan, D. B. Conservative and compensatory evolution in oxidative phosphorylation complexes of angiosperms with highly divergent rates of mitochondrial genome evolution. Evolution 69, 3069-3081, https://doi. org/10.1111/evo.12808 (2015).

33. Schmidt, T. R., Wu, W., Goodman, M. \& Grossman, L. I. Evolution of nuclear- and mitochondrial-encoded subunit interaction in cytochrome c oxidase. Mol. Biol. Evol 18, 563-569, https://doi.org/10.1093/oxfordjournals.molbev.a003836 (2001).

34. Osada, N. \& Akashi, H. Mitochondrial-nuclear interactions and accelerated compensatory evolution: evidence from the primate cytochrome C oxidase complex. Mol. Biol. Evol 29, 337-346, https://doi.org/10.1093/molbev/msr211 (2012).

35. Sloan, D. B., Triant, D. A., Wu, M. \& Taylor, D. R. Cytonuclear interactions and relaxed selection accelerate sequence evolution in organelle ribosomes. Mol. Biol. Evol 31, 673-682, https://doi.org/10.1093/molbev/mst259 (2014).

36. Zhang, J., Ruhlman, T. A., Sabir, J., Blazier, J. C. \& Jansen, R. K. Coordinated rates of evolution between interacting plastid and nuclear genes in Geraniaceae. Plant Cell 27, 563-573, https://doi.org/10.1105/tpc.114.134353 (2015).

37. Maier, U. G. et al. Massively convergent evolution for ribosomal protein gene content in plastid and mitochondrial genomes. Genome Biol. Evol 5, 2318-2329, https://doi.org/10.1093/gbe/evt181 (2013).

38. Rockenbach, K. et al. Positive selection in rapidly evolving plastid-nuclear enzyme complexes. Genet. 204, 1507-1522, https://doi. org/10.1534/genetics.116.188268 (2016).

39. Neuhaus, H. \& Link, G. The chloroplast tRNALys(UUU) gene from mustard (Sinapis alba) contains a class II intron potentially coding for a maturase-related polypeptide. Curr. Genet. 11, 251-257, https://doi.org/10.1007/bf00355398 (1987).

40. Mohr, G., Perlman, P. S. \& Lambowitz, A. M. Evolutionary relationships among group II intron-encoded proteins and identification of a conserved domain that may be related to maturase function. Nucleic Acids Res 21, 4991-4997, https://doi.org/10.1093/ nar/21.22.4991 (1993).

41. Zoschke, R. et al. An organellar maturase associates with multiple group II introns. Proc. Natl Acad. Sci. U.S.A. 107, 3245-3250, https://doi.org/10.1073/pnas.0909400107 (2010).

42. McNeal, J. R., Kuehl, J. V., Boore, J. L., Leebens-Mack, J. \& dePamphilis, C. W. Parallel loss of plastid introns and their maturase in the genus Cuscuta. PLoS One 4, e5982, https://doi.org/10.1371/journal.pone.0005982 (2009).

43. Funk, H. T., Berg, S., Krupinska, K., Maier, U. G. \& Krause, K. Complete DNA sequences of the plastid genomes of two parasitic flowering plant species, Cuscuta reflexa and Cuscuta gronovii. BMC Plant Biol. 7, 45, https://doi.org/10.1186/1471-2229-7-45 (2007).

44. Delannoy, E., Fujii, S., Colas des Francs-Small, C., Brundrett, M. \& Small, I. Rampant gene loss in the underground orchid Rhizanthella gardneri highlights evolutionary constraints on plastid genomes. Mol. Biol. Evol. 28, 2077-2086, https://doi. org $/ 10.1093 / \mathrm{molbev} / \mathrm{msr} 028$ (2011)

45. Birky, C. W. Jr. The inheritance of genes in mitochondria and chloroplasts: laws, mechanisms, and models. Annu. Rev. Genet. 35 , 125-148, https://doi.org/10.1146/annurev.genet.35.102401.090231 (2001).

46. McCauley, D. E. \& Olson, M. S. Associations among cytoplasmic molecular markers, gender, and components of fitness in Silene vulgaris, a gynodioecious plant. Mol. Ecol. 12, 777-787, https://doi.org/10.1046/j.1365-294x.2003.01764.x (2003).

47. McCauley, D. E., Smith, R. A., Lisenby, J. D. \& Hsieh, C. The hierarchical spatial distribution of chloroplast DNA polymorphism across the introduced range of Silene vulgaris. Mol. Ecol. 12, 3227-3235, https://doi.org/10.1046/j.1365-294x.2003.01992.x (2003).

48. Massouh, A. et al. Spontaneous chloroplast mutants mostly occur by replication slippage and show a biased pattern in the plastome of Oenothera. Plant Cell 28, 911-929, https://doi.org/10.1105/tpc.15.00879 (2016).

49. Hirao, T., Watanabe, A., Kurita, M., Kondo, T. \& Takata, K. A frameshift mutation of the chloroplast mat $K$ coding region is associated with chlorophyll deficiency in the Cryptomeria japonica virescent mutant Wogon-Sugi. Curr. Genet. 55, 311-321, https://doi. org/10.1007/s00294-009-0247-9 (2009).

50. Zerbino, D. R. \& Birney, E. Velvet: algorithms for de novo short read assembly using de Bruijn graphs. Genome Res. 18, 821-829, https://doi.org/10.1101/gr.074492.107 (2008).

51. Park, S., An, B. \& Park, S. Reconfiguration of the plastid genome in Lamprocapnos spectabilis: IR boundary shifting, inversion, and intraspecific variation. Sci. Rep. 8, 13568, https://doi.org/10.1038/s41598-018-31938-w (2018)

52. Kearse, M. et al. Geneious Basic: an integrated and extendable desktop software platform for the organization and analysis of sequence data. Bioinformatics 28, 1647-1649, https://doi.org/10.1093/bioinformatics/bts199 (2012).

53. Lowe, T. M. \& Eddy, S. R. tRNAscan-SE: a program for improved detection of transfer RNA genes in genomic sequence. Nucleic Acids Res 25, 955-964 (1997).

54. Laslett, D. \& Canback, B. ARAGORN, a program to detect tRNA genes and tmRNA genes in nucleotide sequences. Nucleic Acids Res 32, 11-16, https://doi.org/10.1093/nar/gkh152 (2004).

55. Greiner, S., Lehwark, P. \& Bock, R. OrganellarGenomeDRAW (OGDRAW) version 1.3.1: expanded toolkit for the graphical visualization of organellar genomes. Nucleic Acids Res. 47, W59-W64, https://doi.org/10.1093/nar/gkz238 (2019).

56. Katoh, K., Misawa, K., Kuma, K.-I. \& Miyata, T. MAFFT: a novel method for rapid multiple sequence alignment based on fast Fourier transform. Nucleic Acids Res 30, 3059-3066 (2002).

57. Nguyen, L.-T., Schmidt, H. A., von Haeseler, A. \& Minh, B. Q. IQ-TREE: a fast and effective stochastic algorithm for estimating maximum-likelihood phylogenies. Mol. Biol. Evol. 32, 268-274, https://doi.org/10.1093/molbev/msu300 (2015).

58. Minh, B. Q., Nguyen, M. A. T. \& von Haeseler, A. Ultrafast approximation for phylogenetic bootstrap. Mol. Biol. Evol. 30, 1188-1195, https://doi.org/10.1093/molbev/mst024 (2013).

59. Bouckaert, R. et al. BEAST 2: A Software Platform for Bayesian Evolutionary. Analysis. PLOS Computational Biol. 10, e1003537, https://doi.org/10.1371/journal.pcbi.1003537 (2014). 
60. Wang, W. et al. The rise of angiosperm-dominated herbaceous floras: Insights from Ranunculaceae. Sci. Rep. 6, 27259, https://doi. org/10.1038/srep27259 (2016).

61. Ghawana, S. et al. An RNA isolation system for plant tissues rich in secondary metabolites. BMC Res. Notes 4 , 85, https://doi. org/10.1186/1756-0500-4-85 (2011)

62. Grabherr, M. G. et al. Full-length transcriptome assembly from RNA-Seq data without a reference genome. Nat. Biotechnol. 29, 644-652, https://doi.org/10.1038/nbt.1883 (2011).

63. Camacho, C. et al. BLAST+: architecture and applications. BMC Bioinformatics 10, 421, https://doi.org/10.1186/1471-2105-10-421 (2009).

64. Emanuelsson, O., Brunak, S., von Heijne, G. \& Nielsen, H. Locating proteins in the cell using TargetP, SignalP and related tools. Nat. Protoc. 2, 953-971, https://doi.org/10.1038/nprot.2007.131 (2007).

65. Marchler-Bauer, A. et al. CDD: conserved domains and protein three-dimensional structure. Nucleic Acids Res. 41, D348-352, https://doi.org/10.1093/nar/gks1243 (2013).

66. Allen, G. C., Flores-Vergara, M. A., Krasynanski, S., Kumar, S. \& Thompson, W. F. A modified protocol for rapid DNA isolation from plant tissues using cetyltrimethylammonium bromide. Nat. Protoc. 1, 2320-2325, https://doi.org/10.1038/nprot.2006.384 (2006).

67. Untergasser, A. et al. Primer3-new capabilities and interfaces. Nucleic Acids Res. 40, e115, https://doi.org/10.1093/nar/gks596 (2012).

68. Huson, D. H. \& Bryant, D. Application of phylogenetic networks in evolutionary studies. Mol. Biol. Evol. 23, 254-267, https://doi. org $/ 10.1093 / \mathrm{molbev} / \mathrm{msj} 030$ (2006).

69. Bryant, D. \& Moulton, V. Neighbor-Net: an agglomerative method for the construction of phylogenetic networks. Mol. Biol. Evol. 21, 255-265, https://doi.org/10.1093/molbev/msh018 (2004).

70. Zmasek, C. M. \& Eddy, S. R. A simple algorithm to infer gene duplication and speciation events on a gene tree. Bioinformatics 17, 821-828, https://doi.org/10.1093/bioinformatics/17.9.821 (2001).

71. Kumar, S., Stecher, G., Li, M., Knyaz, C. \& Tamura, K. MEGA X: molecular evolutionary genetics analysis across computing platforms. Mol. Biol. Evol. 35, 1547-1549, https://doi.org/10.1093/molbev/msy096 (2018).

72. Yang, Z. PAML 4: phylogenetic analysis by maximum likelihood. Mol. Biol. Evol. 24, 1586-1591, https://doi.org/10.1093/molbev/ msm088 (2007)

73. R: A Language and Environment for Statistical Computing. (The R Foundation for Statistical Computing, Vienna, Austria, 2015).

74. Edgar, R. C. MUSCLE: a multiple sequence alignment method with reduced time and space complexity. BMC Bioinformatics 5, 113, https://doi.org/10.1186/1471-2105-5-113 (2004)

\section{Acknowledgements}

We thank Kae Sun Chang at the Korea National Arboretum for providing the herbarium specimens. This work was supported by the 2017 Yeungnam University Grant (217A380095), South Korea.

\section{Author contributions}

S.P. contributed to the design of the project; assembled, finished, and annotated the plastid genome; performed all analyses; prepared the figures and tables; and drafted the manuscript. B.A. designed and performed experiments and read/edited the manuscript. S.J.P. contributed to the design of the project and read/edited the manuscript. All authors read and approved the final draft of the manuscript.

\section{Competing interests}

The authors declare no competing interests.

\section{Additional information}

Supplementary information is available for this paper at https://doi.org/10.1038/s41598-020-59547-6.

Correspondence and requests for materials should be addressed to S.J.P.

Reprints and permissions information is available at www.nature.com/reprints.

Publisher's note Springer Nature remains neutral with regard to jurisdictional claims in published maps and institutional affiliations.

Additional Information

(c) (i) Open Access This article is licensed under a Creative Commons Attribution 4.0 International

License, which permits use, sharing, adaptation, distribution and reproduction in any medium or format, as long as you give appropriate credit to the original author(s) and the source, provide a link to the Creative Commons license, and indicate if changes were made. The images or other third party material in this article are included in the article's Creative Commons license, unless indicated otherwise in a credit line to the material. If material is not included in the article's Creative Commons license and your intended use is not permitted by statutory regulation or exceeds the permitted use, you will need to obtain permission directly from the copyright holder. To view a copy of this license, visit http://creativecommons.org/licenses/by/4.0/.

(c) The Author(s) 2020 